Research Paper

\title{
A Randomized Controlled Study of rhTPO and rhIL-11 for the Prophylactic Treatment of Chemotherapy- Induced Thrombocytopenia in Non-Small Cell Lung Cancer
}

\author{
Yunhua $\mathrm{Xu}^{1}$, Xia Song ${ }^{2}$, Fangmin $\mathrm{Du}^{3}$, Qiong $\mathrm{ZhaO}^{4}$, Li Liu ${ }^{5}$, Zhiyong Ma ${ }^{6}$, Shun $\mathrm{Lu}^{1}{ }^{\circledR}$ \\ 1. Department of Shanghai Lung Cancer Center, Shanghai Chest Hospital, Shanghai Jiao Tong University, No.241West Huaihai Road, Shanghai, P. R. China. \\ 2. Shanxi Cancer Hospital, No.309 West Yanta Road, Shanxi, P. R. China. \\ 3. Dongyang people's Hospital of Zhejiang Province, No.60 West Wuning Road, Dongyang, P. R. China. \\ 4. The First Affiliated Hospital of Zhejiang University, No. 79 Qingchun Road, Hangzhou, P. R. China \\ 5. The Affilicated Wuhan Union Hospital of Huazhong University of Science and Technology, No.1277 Liberation Avenue, Wuhan, P. R. China. \\ 6. Henan Cancer Hospital, No.127 Dongming Road, Zhengzhou, P. R. China.
}

$\triangle$ Corresponding author: Shun Lu, M.D., Ph.D. Department of Shanghai Lung Cancer Center, Shanghai Chest Hospital, Shanghai Jiao Tong University, 241 Huaihai West Road, Shanghai 200030, China. Tel.: +862122200000; Fax: +862162804970. E-mail: shunlu@sjtu.edu.cn

(C) Ivyspring International Publisher. This is an open access article distributed under the terms of the Creative Commons Attribution (CC BY-NC) license (https://creativecommons.org/licenses/by-nc/4.0/). See http://ivyspring.com/terms for full terms and conditions.

Received: 2018.04.15; Accepted: 2018.08.28; Published: 2018.11.25

\begin{abstract}
Background: rhTPO and rhlL-11 are both recommended for the prophylactic treatment of chemotherapy-induced thrombocytopenia (CIT). However, there has been no head to head comparative study on the prophylactic administration of rhTPO and rhlL-11 to alleviate CIT in non-small cell lung cancer (NSCLC).

Methods: In this open-label prospective multi-center phase II clinical trial, 108 NSCLC patients who experienced severe CIT after prior chemotherapy were randomized into study and control arms. Patients in the study arm were prophylactically administered rhTPO on day 2, day 4, day 6 and day 9 of the subsequent chemotherapy cycle, while patients in the control arm accepted prophylactic rhlL-11 from day 9 to day 15 of the subsequent chemotherapy cycle.

Results: During the trial, the median time required for recovery of the platelet count to $\geq 75 \times 109 / \mathrm{L}$ was 3 days (range: $2-4$ ) in the study arm and 4 days (range: $2-6)$ in the control arm $(P=0.398)$. The lowest platelet counts were $61.8 \pm 39.9 \times 10 \% / \mathrm{L}$ in the study arm, values higher than those measured in the control arm $52.8 \pm 36.8 \times 10 \% / \mathrm{L}(P=1.044)$. Platelet counts $<50 \times 10 \% / \mathrm{L}$ occurred in $46.2 \%$ of patients in the study arm vs $58.6 \%$ in the control arm $(P=0.368)$. There were no drug-related adverse reactions in the study arm, but 4 cases $(12.9 \%)$ in the control arm $(P=0.008)$, especially cardiotoxicity $(P=0.022)$.

Conclusion: Prophylactic administration of rhTPO helps to alleviate CIT in NSCLC as well as rhIL-11, but is safer to use and more convenient to administer.
\end{abstract}

Key words: thrombocytopenia, rhTPO, rhIL-11, prophylactic administration, NSCLC

\section{Introduction}

Lung cancer is a very common form of malignancy with a high incidence and mortality, particularly in heavy smokers [1]. First-line chemotherapy is one of the main treatments for advanced non-small cell lung cancer (NSCLC), including platinum with third generation anticancer drugs (paclitaxel, gemcitabine, pemetrexed, etc.) [2-4]. In addition, gemcitabine combined with platinum (cisplatin or carboplatin) is commonly used because of its wide range of applications and low cost. However, it is the most common chemotherapy regimen that causes chemotherapy-induced thrombocytopenia (CIT) in NSCLC patients, with the highest incidence occurring in III or IV-degree CIT [5]. 
Severe CIT usually leads to a dose reduction or interruption of treatment, and thus affects the clinical efficacy and patient survival rate [6,7]. Platelet transfusion remains the fastest and most effective treatment for severe CIT, but repeated platelet transfusion induces platelet antibody formation rendering eventually the treatment ineffective [8]. First-generation recombinant thrombopoietins research has been halted due to antibody formation against endogenous thrombopoietin [9]. The China Food and Drug Administration (CFDA) to date have not formally approved a second-generation thrombopoietin receptor agonist. According to the Expert Consensus on Diagnosis and Treatment of Chemotherapy-Induced Thrombocytopenia in Cancer Patients in China, rhIL-11 was recommended as prophylactic treatment 6-24 h after chemotherapy for those patients who experienced III or IV-degree CIT in a prior cycle or were at a high risk of bleeding [10]. Nevertheless, adverse reactions occur at an unacceptable frequency, especially cardiotoxicity, which limits its use in clinical practice [11,12]. rhTPO is recommended to be administered early in patients with III or IV-degree CIT in a prior cycle of chemotherapy or if they were at a high risk of bleeding [13].

In a small sample retrospective study, we found that the short-term intermittent prophylactic administration of rhTPO could alleviate CIT admirably in NSCLC [14]. However, there has been no comparative study on the prophylactic administration of rhTPO and rhIL-11 to alleviate CIT in patients with NSCLC. Therefore, we designed a prospective randomized controlled multi-center clinical trial to compare rhTPO with rhIL-11 for the prevention of serious CIT in patients with NSCLC.

\section{Materials and Methods}

\section{Study population}

The clinical trial was designed as a prospective, open-label, randomized, non-inferiority, multicenter study and performed synchronously in multiple centers (16 centers) from May 2009 to June 2015. All patients voluntarily signed informed consent forms. This study met the basic standards of the Declaration of Helsinki and was approved by the Institutional Review Board of each center (The ClinicalTrials.gov Identifier: NCT02344979). The protocols for our study were approved by the Institutional Review Board of Shanghai Chest Hospital (ethical approval number: 200811I-06).

\section{Inclusion criteria}

- Patients aged from 18 to 75 years, both male and female.
- NSCLC identified by either histology or cytology.

- Compliance with indications of chemotherapy (white blood cell $\geq 4.0 \times 10^{9} / \mathrm{L}$; neutrophil count $>$ $1.5 \times 10^{9} / \mathrm{L}$; platelet counts $\geq 100 \times 10^{9} / \mathrm{L}$; alanine aminotransferase $\leq 3$ times higher than the normal upper limit; aspartate transaminase $\leq 3$ times higher than the normal upper limit; total bilirubin $\leq$ twice the normal upper limit; Eastern Cooperative Oncology Group performance status score (ECOG PS) $\leq 2$ points; without severe cardio-respiratory dysfunction).

- Occurrence of platelet reduction in the chemotherapy cycle, platelet $\leq 50 \times 10^{9} / \mathrm{L}$.

- Expected survival time $\geq 3$ months.

- The patient could understand he study aims and was willing and to sign an informed consent form.

- Compliance with the research and follow-up program.

\section{Exclusion criteria}

- Pregnant or lactating women.

- History of severe allergy to biological agents.

- Existing severe acute infection that was not controlled.

- Patients receiving radiotherapy.

- Three or more sites of osseous metastasis during screening and platelet counts $<100 \times 10^{9} / \mathrm{L}$ on day 17 of the prior chemotherapy cycle.

- Patients having a previous history of pulmonary artery embolism or myocardial infarction, or a history of thrombus or moveable thrombus in the previous 3 months.

- Onset of septicemia, disseminated intravascular coagulation (DIC), hypersplenism, or any other known condition that may have aggravated the thrombocytopenia during past cycle(s) of chemotherapy.

- Patients with central nervous system (CNS) metastasis.

\section{Withdrawal criteria}

- The subject requested to withdraw from the trial for any reason.

- Occurrence of intolerable adverse reactions.

- Bad compliance and affected efficacy caused by failure to comply with the specified administration scheme. 
- The research and observation could not continue due to some unpredictable situation during the trial.

\section{Number of designed samples}

Altogether 120 samples and a ratio of 2:1 for the study arm $(n=80)$ and control arm $(n=40)$ were designed for the trial.

\section{Therapeutic scheme}

\section{Drugs under research}

Recombination human thrombopoietin (rhTPO) injection (TPIAO, Shenyang 35 BIO Incorporation, $15,000 \mathrm{U} / \mathrm{mL} /$ ampoule), and recombinant human interleukin-11 (rhIL-11) injection (Juheli, Qilu Pharmaceutical Co., Ltd, $3.0 \mathrm{mg} /$ ampoule).

\section{Routes of administration}

Lung cancer patients who had platelet counts $\leq$ $50 \times 10^{9} /$ L after prior chemotherapy with gemcitabine /cisplatin (GC) or gemcitabine/ carboplatin (GP) were admitted and randomized into trial and control arms.

\section{Study arm}

The patients accepted subcutaneous injection of 15,000 U rhTPO on day 2, day 4, day 6 and day 9 of the chemotherapy cycle. Details are shown in Fig. 1.

\section{Control arm}

The patients accepted a subcutaneous injection of $3 \mathrm{mg}$ rhIL-11 for 7 consecutive days from day 9 to day 15. The details are shown in Fig. 1.

\section{Chemotherapy regimen}

GP regimen: $1,250 \mathrm{mg} / \mathrm{m}^{2}$ gemcitabine on day 1 and day $8 ; 75 \mathrm{mg} / \mathrm{m}^{2}$ cisplatin on day 1 or divided into 3 days; $\mathrm{q} 21 \mathrm{~d}$.

GC regimen: $1,250 \mathrm{mg} / \mathrm{m}^{2}$ gemcitabine on day 1 and day $8 ; A U C=5 \mathrm{mg} / \mathrm{mL} / \mathrm{min}$ carboplatin on day $1 ;$ q21d.
If in the previous cycle, there were platelet counts $<25 \times 10^{9} / \mathrm{L}$, and bleeding symptoms (oral mucosa, gingival bleeding, or skin petechia), the dose administered in the subsequent chemotherapy cycle was reduced by $25 \%$.

\section{Drug-withdrawal indications}

During the administration of rhTPO and rhIL-11, it was withdrawn when the platelet counts increased to $100 \times 10^{9} / \mathrm{L}$ or the increment was greater than $\geq 50$ $\times 10^{9} / \mathrm{L}$.

\section{Supplemental therapy}

Platelet transfusion: (1) when the platelet counts were $\leq 10 \times 10^{9} / \mathrm{L}$, single-donor platelets would be suggested for transfusion; (2) when $10 \times 10^{9} / \mathrm{L}<$ platelet counts $<25 \times 10^{9} / \mathrm{L}$ and the patient had a high risk of bleeding, single-donor platelets were considered for transfusion; (3) when $25 \times 10^{9} / \mathrm{L} \leq$ platelet counts $<50 \times 10^{9} / \mathrm{L}$, the treatment decision was decided by the physician in charge.

\section{Efficacy and safety evaluation}

\section{Efficacy evaluation}

Primary efficacy indicator

Days required for the recovery of platelet to $\geq 75$ $\times 10^{9} / \mathrm{L}$;

\section{Secondary efficacy indicators}

- Lowest value, highest recovered value after treatment and the platelet counts before and after treatment in the 2 arms

- Days of platelet counts $<50 \times 10^{9} / \mathrm{L}$;

- Days required for the recovery of platelet levels to $\geq 100 \times 10^{9} / \mathrm{L}$ and increments to $>50 \times 10^{9} / \mathrm{L}$

- Platelet counts on day 21 in the 2 arms Platelet transfusion frequency and amount

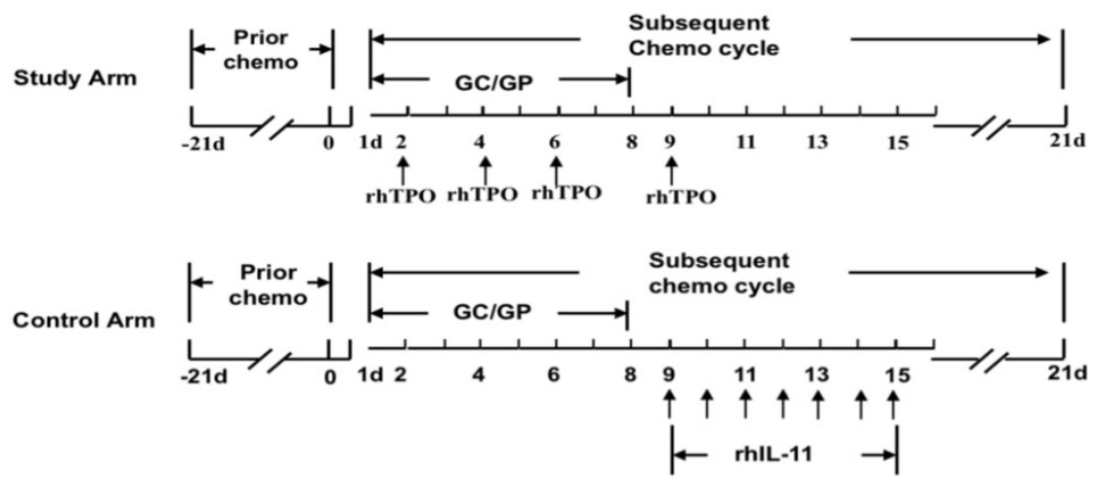

Figure 1. Routes of administration in two arms. Patients when had platelet counts $\leq 50 \times 109 / \mathrm{L}$ in the prior chemotherapy cycle were enrolled into the current study. The patients in the study arm would accept subcutaneous injection of 15,000 $\mathrm{U}$ rhTPO on $\mathrm{d} 2$, d4, d6 and d9 of the subsequent chemotherapy cycle. The patients in the control arm would accept subcutaneous injection of $3 \mathrm{mg}$ rhlL-11 for 7 days from d9 to d15 of the subsequent chemotherapy cycle. 


\section{Safety evaluation}

Routine blood examinations, urinalysis, hepatorenal function, coagulation function, electrocardiograph (ECG), vital signs, and adverse events (frequency, severity, duration, and classification) in the 2 arms were compared.

\section{Statistical methods}

Continuous variables were adopted to determine the number, mean value, standard deviation, median, maximum and minimum values, and counts to establish the frequency and constituent ratio.

\section{Analysis of the primary efficacy indicator}

- Days required for the recovery of platelet levels to $\geq 75 \times 10^{9} / \mathrm{L}$ in the 2 arms: Cox regression analysis, with the baseline of the platelet count as a concomitant variable, was employed to compute the hazard ratios of the 2 arms and the 95\% confidence interval (CI).

- Subsequently, the days required for the recovery of platelet levels to $\geq 75 \times 10^{9} / \mathrm{L}$ were analyzed using the Kaplan-Meier method, 25\%, 50\% and $75 \%$ quantiles of the days required for recovery of platelet level in the 2 arms to $\geq 75 \times 10^{9} / \mathrm{L}$ and the corresponding $95 \% \mathrm{CI}$ were documented respectively, and comparisons between the 2 arms were verified using a log-rank test.

\section{Analysis of secondary efficacy indicators}

- The inter-arm comparison of the lowest platelet level, highest platelet level after treatment, and changes in platelet levels before and after the treatment in the 2 arms was compared using a $t$-test.

- The days during which the platelet count was < $50 \times 10^{9} / \mathrm{L}$ were analyzed using the KaplanMeier method, and the median time and $95 \% \mathrm{CI}$ of the 2 arms were determined. A log-rank test was employed to detect any differences between the 2 arms.

- The days required for the recovery of platelet levels to $\geq 100 \times 10^{9} / \mathrm{L}$ and an increment by $>50$ $\times 10^{9} / \mathrm{L}$ were analyzed with the Kaplan-Meier method. The median time and $95 \% \mathrm{CI}$ of the 2 arms were listed respectively, and a log-rank test was employed to look for the differences between the 2 arms.

- The differences in platelet counts on day 21 between the 2 arms were compared using a $t$-test.

- The inter-arm differences in the platelet transfusion frequency and amounts administered were compared using a rank sum test.

\section{Safety analysis}

- The incidence of adverse events and adverse drug reactions was determined by the investigator and the severity of adverse events classified according to the National Cancer Center Common Toxicity Criteria (NCI-CTC, ver. 3.0). Adverse events refer to adverse clinical signs that occurred during drug treatment. Adverse reactions refer to the adverse effects of a specific drug that is unrelated to its intended or accidental use in normal clinical practice.

Statistical software and general requirements

- SPSS ver. 23.0 software was used for analysis purposes.

- All the statistical tests used were two-sided, and $P<0.05$ was deemed to be statistically significant.

\section{Results}

\section{General clinical materials}

A total of 108 NSCLC patients suffering from CIT after receiving gemcitabine in combination with cisplatin or carboplatin chemotherapy were enrolled and randomized into a study arm and control arm. There were 77 cases in the study arm and 31 in the control arm. A total of 55 cases received GC and 53 GP. There were no statistically significance differences in age, height, weight, or sex $(P>0.05)$ between the arms of patients. A pre-chemotherapy comparison of all the indicators of vital signs and blood routine examinations in the 2 arms revealed no statistically significant differences $(P>0.05)$, while a comparison of the chemotherapy regimens during the treatment also failed to find a statistically significant difference $(P>0.05$, Table 1$)$.

\section{Efficacy analysis}

\section{Days for platelet levels to recover to $\geq 75 \times 10 \% / \mathrm{L}$}

The median days for the recovery of platelet levels to $\geq 75 \times 10^{9} / \mathrm{L}$ was 3 (range: $2-4$ ) in the study arm and 4 (range: $2-6)$ in the control arm $(P=0.398)$, but the difference was not statistically significant $(P>$ $0.05)$. The recovery rate of platelets to $>75 \times 10^{9} / \mathrm{L}$ revealed that more patients in the study arm recovered $(84.4 \%$ vs $77.4 \%)$ within a short period of time (Fig. 2).

\section{Platelet changing tendency in the 2 arms}

The lowest platelet level $\left(\times 10^{9} / \mathrm{L}\right)$ in the study arm was $61.8 \pm 39.9$ compared with $52.8 \pm 36.8$ in the control arm $(P=0.299)$. The highest platelet counts $(x$ $10^{9} / \mathrm{L}$ ) in the study arm was $223.5 \pm 127.3$ compared with $245.8 \pm 158.7$ in the control arm $(P=0.463)$. The 
increment between the highest value and baseline value of platelet counts $\left(\times 10^{9} / \mathrm{L}\right)$ in the study arm was $26.1 \pm 166.8$ compared with $22.2 \pm 200.6(P=$ 0.487 ), and no statistical significance was found using a $t$-test (Fig. 3).

\section{Days of platelet counts $<50 \times 10^{9} / \mathrm{L}$}

The median days for the recovery of platelet levels to $<50 \times 10^{9}$ / L was 2 (range: $0-4$ ) in the study arm and 2 (range: $0-5)$ in the control arm $(P=0.691)$, but the difference was not statistically significant $(P>$ $0.05)$. According to the comparison of the 2 arms in terms of days of platelet counts $<50 \times 10^{9} / \mathrm{L}$, the ratio of patients with platelet counts $<50 \times 10^{9} / \mathrm{L}$ was $46.2 \%$ in the study arm vs $58.6 \%$ in the control arm, but there was no statistically significant difference between the 2 arms ( $P=0.446$, Fig. 4$)$.

\section{Days for platelet recovery to $\geq 100 \times 10 \% / \mathrm{L}$}

The time in days for the platelet levels to recover to $\geq 100 \times 10^{9} / \mathrm{L}$ in the study arm was 6 (range: $4-7$ ) compared with 6 days (range: 4-8) in the control arm $(P=0.734)$, but the difference was not statistically significant $(P>0.05)$.

The platelet count in the study arm was $181.2 \pm$ $102.7 \times 10^{9} / \mathrm{L}$ compared to $224.5 \pm 161 \times 10^{9} / \mathrm{L}$ in the control arm on day $21(P=0.263)$. After prophylactic treatment for CIT, there were no interruptions in the subsequent chemotherapy cycles in either arm.

\section{Platelet transfusion}

In the study arm, 3 patients $(4.5 \%)$ required transfusion of $1 \mathrm{u}$ platelets and 2 patients in the control arm (7.4\%). After analysis using a continuous chi-squared correction test, no statistically significant difference was found between the 2 arms $(P=0.948$, Table 2).

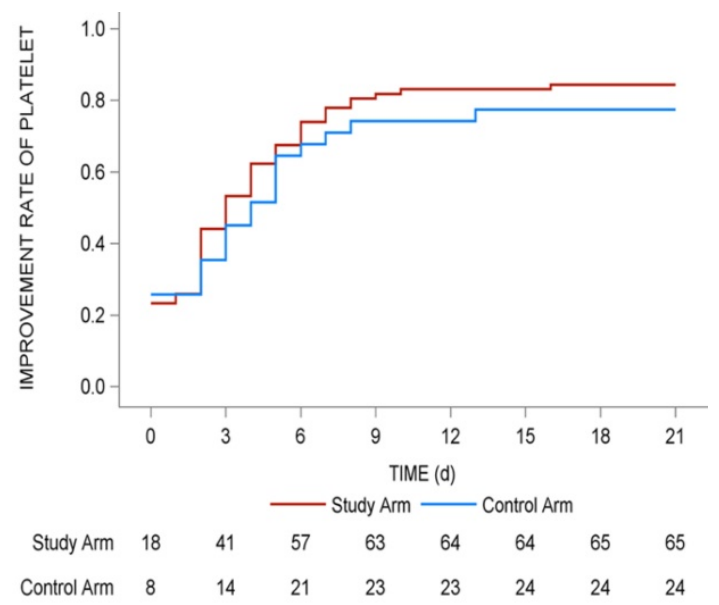

Figure 2. Improvement rate of platelet in whole 21 days of the subsequent chemotherapy cycle. The improvement rates of platelet in whole 21 days of the subsequent chemotherapy cycle were shown with red line (study arm) and blue line (control arm). The number of patients in two arms with recovery of platelets $>75 \times 109 / \mathrm{L}$ in different days were shown under the lines.

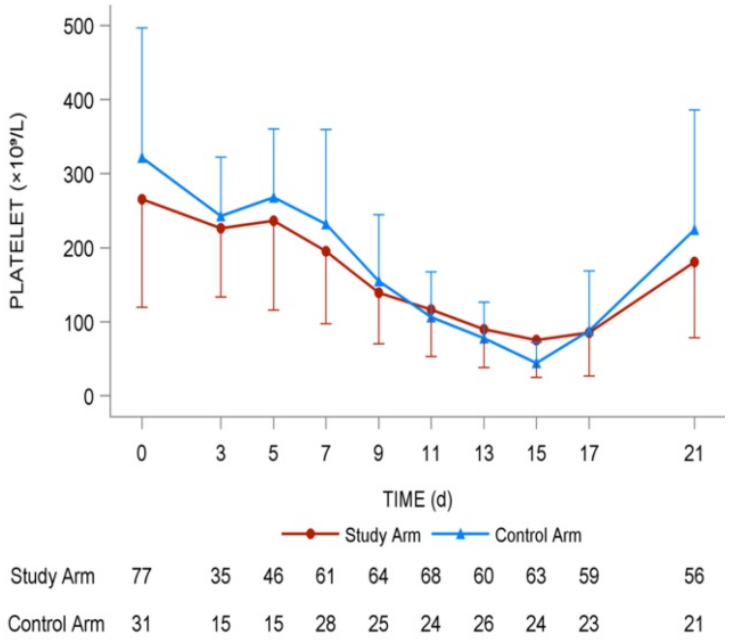

Figure 3. Dynamic platelet changing tendency in the two arms. It was mapped with red line (study arm) and blue line (control arm). The number of patients in two arms with different platelets levels were shown under the lines.

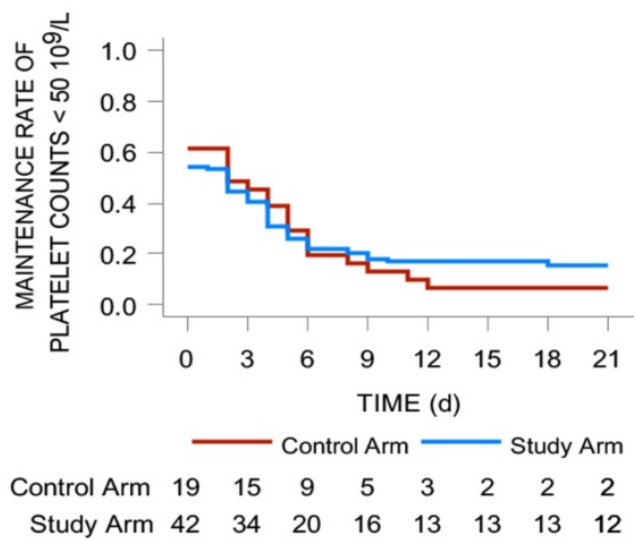

Figure 4. Maintenance rate of platelet counts $<50 \times 109 / \mathrm{L}$ in the two arms. It was mapped with red line (study arm) and blue line (control arm). The number of patients in two arms with different platelets levels were shown under the lines.

\section{Analysis of the area under the curve (AUC) of platelet counts}

During the cycle of chemotherapy from day 1 to day 21, no significant difference was detected in the platelet counts in either the trial or control arms (Fig. 5). But from day 9 to day 17, the lowest platelet level in the study arm was higher than in the control arm, and the platelet count AUC of the study arm was $>$ than that of the control arm $(P>0.05)$.

\section{Safety analysis}

\section{Adverse events and adverse drug reactions}

During the trial, a total of 37 adverse events were recorded. The occurrence of adverse events in the study arm was $25(32.5 \%)$ compared with 12 (38.7\%) in the control arm $(P>0.05$, Table 3$)$. No severe adverse events occurred in either arm during the trial. 
The study arm had no adverse drug reactions while the incidence of adverse reactions in the control arm was 4 (12.9\%). A comparison between them revealed a statistically significant difference $(P=$ 0.008 , Table 4). There were 2 patients with cardiotoxicity, 1 patient with dizziness, 1 with limb edema, which was related to rhIL-11. There was a significant difference only in comparison of cardiotoxicity (0 vs 9.7\%, $P=0.022$ ) while other adverse reactions were not different.

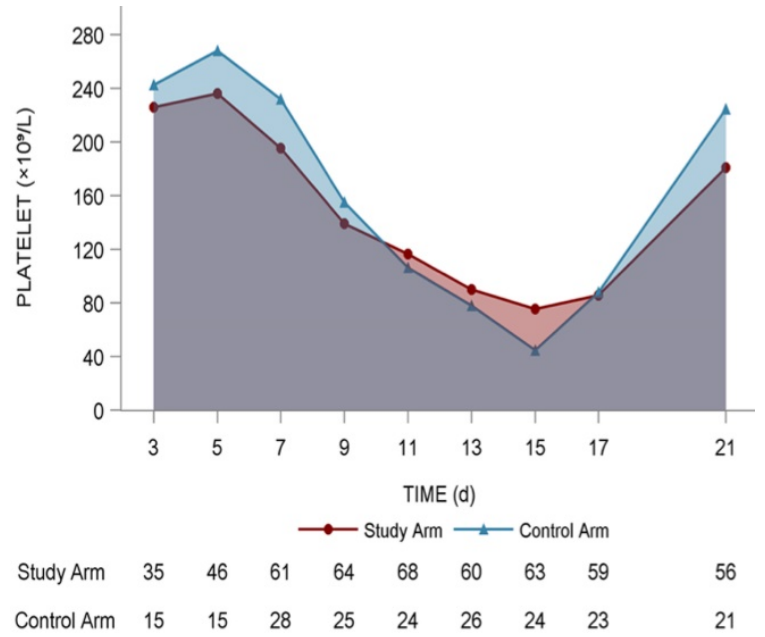

Figure 5. The area under the curve (AUC) of platelet counts in whole 21 days of the subsequent chemotherapy cycle. The curve (AUC) of platelet counts in whole 21 days of the subsequent chemotherapy cycle were shown with red line (study arm) and blue line (control arm). The number of patients in two arms with different platelets levels were shown under the lines.

Table 1. General data between the study arm and the control arm

\begin{tabular}{|c|c|c|c|c|}
\hline Features & Study arm & Control arm & P-value & Test \\
\hline Age (years) & & & 0.274 & $\mathrm{t}$ \\
\hline $\begin{array}{l}\text { Mean } \pm \text { SD } \\
\text { (Range) }\end{array}$ & $\begin{array}{l}58.7 \pm 8.9 \\
(30.0 \sim 75.0)\end{array}$ & $\begin{array}{l}60.8 \pm 7.6 \\
(38.0 \sim 75.0)\end{array}$ & & \\
\hline Height (cm) & & & 0.896 & $\mathrm{t}$ \\
\hline $\begin{array}{l}\text { Mean } \pm \text { SD } \\
\text { (Range) }\end{array}$ & $\begin{array}{l}165.1 \pm 7.2 \\
(148.0 \sim 180.0)\end{array}$ & $\begin{array}{l}164.9 \pm 8.0 \\
(161.6 \sim 168.2)\end{array}$ & & \\
\hline Weight (kg) & & & 0.423 & $\mathrm{t}$ \\
\hline $\begin{array}{l}\text { Mean } \pm \text { SD } \\
\text { (Range) }\end{array}$ & $\begin{array}{l}61.7 \pm 10.4 \\
(42.5 \sim 85.0)\end{array}$ & $\begin{array}{l}59.8 \pm 10.5 \\
(41.0 \sim 91.0)\end{array}$ & & \\
\hline Gender & & & 0.138 & Chi-squared \\
\hline $\mathrm{M}(\mathrm{n} / \%)$ & $56(72.7)$ & $18(58.1)$ & & \\
\hline $\mathrm{F}(\mathrm{n} / \%)$ & $21(27.3)$ & $13(41.9)$ & & \\
\hline $\mathrm{T}\left({ }^{\circ} \mathrm{C}\right)$ & $36.6 \pm 0.3$ & $36.6 \pm 0.5$ & 0.558 & $t^{\prime}$ \\
\hline $\mathrm{P}(\mathrm{n} / \mathrm{min})$ & $79.9 \pm 7.2$ & $80.6 \pm 10.8$ & 0.749 & $\mathrm{t}^{\prime}$ \\
\hline $\mathrm{R}(\mathrm{n} / \mathrm{min})$ & $19.1 \pm 1.4$ & $19.3 \pm 1.3$ & 0.482 & $t$ \\
\hline SBP (mmHg) & $118.0 \pm 11.0$ & $119.5 \pm 11.0$ & 0.585 & $\mathrm{t}$ \\
\hline DBP (mmHg) & $73.7 \pm 6.9$ & $74.9 \pm 9.1$ & 0.523 & $\mathrm{t}$ \\
\hline $\mathrm{Hb}(\mathrm{g} / \mathrm{L})$ & $115.29 \pm 17.18$ & $114.97 \pm 17.06$ & 0.931 & $\mathrm{t}$ \\
\hline WBC $(\times 109 / \mathrm{L})$ & $6.24 \pm 3.07$ & $6.51 \pm 2.77$ & 0.678 & $t$ \\
\hline PLT (× 109/L) & $265.27 \pm 145.84$ & $322.06 \pm 174.52$ & 0.087 & $\mathrm{t}$ \\
\hline \multicolumn{3}{|c|}{ Chemotherapy regimen $\mathrm{n}(\%)$} & 0.524 & Chi-squared \\
\hline GC & $38(49.4)$ & $18(58.1)$ & & \\
\hline GP & $39(50.6)$ & $13(41.9)$ & & \\
\hline \multicolumn{3}{|c|}{ Prior cycle platelet transfusion } & 0.971 & Chi-squared \\
\hline Yes & $32(41.6)$ & $13(41.9)$ & & \\
\hline No & $45(58.4)$ & $18(58.1)$ & & \\
\hline
\end{tabular}

Table 2. Infusion frequency and volume of platelets

\begin{tabular}{|c|c|c|c|c|c|}
\hline & $\begin{array}{l}\text { Study arm } \\
\mathrm{N}(\%)\end{array}$ & $\begin{array}{l}\text { Control } \\
\text { arm N (\%) }\end{array}$ & Statistics & P-value & Method \\
\hline \multicolumn{6}{|c|}{ Infusion frequency (times) } \\
\hline 0 times & $64(95.5)$ & $25(92.6)$ & 0.004 & 0.948 & $\begin{array}{l}\text { Chi-squared } \\
\text { test }\end{array}$ \\
\hline 1 times & $3(4.5)$ & $2(7.4)$ & & & \\
\hline Total (missing) & $67(10)$ & $27(4)$ & & & \\
\hline \multicolumn{6}{|c|}{ Infusion volume (u) } \\
\hline $1 \mathrm{u}$ & $3(100.0)$ & $1(50.0)$ & -- & 0.400 & $\begin{array}{l}\text { Fisher exact } \\
\text { test }\end{array}$ \\
\hline $2 u$ & $0(0.0)$ & $1(50.0)$ & & & \\
\hline Total & 3 & 2 & & & \\
\hline
\end{tabular}

Table 3. Comparison of adverse events in the 2 arms

\begin{tabular}{|c|c|c|c|c|}
\hline $\begin{array}{l}\text { Adverse } \\
\text { events }\end{array}$ & $\begin{array}{l}\text { Study arm } \\
(\mathrm{N}=77) \mathrm{n}(\%)\end{array}$ & $\begin{array}{l}\text { Control arm } \\
(\mathrm{N}=31) \mathrm{n}(\%)\end{array}$ & $P$-value & Methods \\
\hline \multicolumn{5}{|c|}{ Myelosuppression (non-thrombocytopenia) } \\
\hline Yes & $19(24.7)$ & $5(16.1)$ & 0.334 & Chi-squared test \\
\hline No & $58(75.3)$ & $26(83.9)$ & & \\
\hline \multicolumn{5}{|c|}{ Gastrointestinal reaction } \\
\hline Yes & $9(11.7)$ & $4(12.9)$ & 1.000 & $\begin{array}{l}\text { Continuous } \\
\text { correction } \\
\text { chi-squared test }\end{array}$ \\
\hline No & $68(88.3)$ & $27(87.1)$ & & \\
\hline \multicolumn{5}{|c|}{ Heart toxicity } \\
\hline Yes & $2(2.6)$ & $4(12.9)$ & 0.099 & $\begin{array}{l}\text { Continuous } \\
\text { correction } \\
\text { chi-squared test }\end{array}$ \\
\hline No & $75(97.4)$ & $27(87.1)$ & & \\
\hline \multicolumn{5}{|l|}{ Fatigue } \\
\hline Yes & $2(2.6)$ & $1(3.2)$ & 1.000 & Fisher \\
\hline No & $75(97.4)$ & $30(96.8)$ & & \\
\hline \multicolumn{5}{|c|}{ Limb edema } \\
\hline Yes & $0(0)$ & $1(3.2)$ & 0.287 & Fisher \\
\hline No & 77 (100) & $30(96.8)$ & & \\
\hline \multicolumn{5}{|c|}{ Dizziness } \\
\hline Yes & $0(0)$ & $1(3.2)$ & 0.287 & Fisher \\
\hline No & 77 (100) & $30(96.8)$ & & \\
\hline \multicolumn{5}{|l|}{ Fever } \\
\hline Yes & $1(1.3)$ & $0(0)$ & 1.000 & Fisher \\
\hline No & $76(98.7)$ & $31(100)$ & & \\
\hline \multicolumn{5}{|l|}{ Cough } \\
\hline Yes & $3(3.9)$ & $0(0)$ & 0.556 & Fisher \\
\hline No & 74 (96.1) & $31(100)$ & & \\
\hline \multicolumn{5}{|l|}{ Others } \\
\hline Yes & $1(1.3)$ & $1(3.2)$ & 0.494 & Fisher \\
\hline No & 76 (98.7) & $30(96.8)$ & & \\
\hline
\end{tabular}

Table 4. Comparison of adverse drug reactions in the 2 arms of patients

\begin{tabular}{lllll}
\hline $\begin{array}{l}\text { Adverse drug } \\
\text { reaction }\end{array}$ & $\begin{array}{l}\text { Study arm } \\
(\mathbf{N}=77) \mathbf{n}(\mathbf{\%})\end{array}$ & $\begin{array}{l}\text { Control arm } \\
(\mathbf{N}=\mathbf{3 1}) \mathbf{n} \mathbf{( \% )}\end{array}$ & P-value & Methods \\
\hline $\begin{array}{l}\text { Heart toxicity } \\
\text { Yes }\end{array}$ & $0(0)$ & $3(9.7)$ & 0.022 & Fisher \\
No & $77(100)$ & $28(90.3)$ & & \\
Limb edema & & $1(3.2)$ & 0.287 & Fisher \\
Yes & $0(0)$ & $30(96.8)$ & & \\
No & $77(100)$ & $1(3.2)$ & 0.287 & Fisher \\
Dizziness & & $30(96.8)$ & & \\
Yes & $0(0)$ & & & \\
No & $77(100)$ & &
\end{tabular}




\section{Discussion}

In clinical practice, thrombopoietin is commonly recommended for the treatment of severe CIT [15]. However, the thrombopoietin effect on platelet numbers usually takes a long time leading to an increased bleeding risk due to thrombopenia. Furthermore, thrombopoietin administration may induce an excessive increase in platelet increments, which also brings the risk of venous thrombosis embolism (VTE). Therefore, the concept of prophylactic administration has been raised in clinical practice. The standard protocol of rhIL-11 administration was recommended for prophylactic administration in 2010 in China while rhTPO was recommended subsequently in 2014 [10].

Vadhan-Raj divided sarcoma patients $(\mathrm{n}=66)$ receiving doxorubicin and ifosfamide (AI regimen) equally into 11 arms (6 patients in each arm), with rhTPO administered at different time points for CIT. It was found that the arms receiving rhTPO on day 5 , -day 3, -day 1 and +day 4 of chemotherapy achieved better platelet recovery compared with the other arms. This result may be due to the fact that the lowest platelet counts appeared about 12 days after the AI regimen [13]. Therefore, the rhTPO administration time depends on the chemotherapy regimen and the time of the platelet nadir. For a short-term chemotherapy regimen and/or the earlier appearance of the platelet nadir, it would be reasonable to begin rhTPO administration shortly after the start of chemotherapy. As reported in previous studies, CIT was commonly started on day 7 after chemotherapy, and reached a nadir on days 10-14 after gemcitabine combined with cisplatin or carboplatin (GC/GP) [17]. Considering these results and the long half-life of rhTPO, we administered rhTPO on day 2, day 4, day 6 and day 9 of the subsequent cycle to NSCLC patients who suffered from III degree or worse CIT in the previous GC/GP cycle, in a small sample experimental study. The results revealed that short-term intermittent prophylactic administration of rhTPO could alleviate the severity of thrombopenia and promote recovery [13]. Therefore, a prospective clinical trial of NSCLC patients who experienced thrombopenia after prior chemotherapy with gemcitabine in combination with cisplatin or carboplatin was performed. Short-term intermittent prophylactic administration of rhTPO was compared with prophylactic administration using rhIL-11 in a standard protocol in terms of its efficacy and adverse drug reactions.

As indicated by the results, in spite of a shorter duration of administration, the study arm showed fewer days for the recovery of platelet levels to $75 \times$ $10^{9} / \mathrm{L}$ and $100 \times 10^{9} / \mathrm{L}$, and the lowest platelet level appeared to be higher than in the control arm. Although the differences were not statistically significant, it was proven that the efficacy of short-term intermittent prophylactic use of rhTPO was similar to the prophylactic administration of rhIL-11. In terms of safety, rhI-11 is contraindicated by the FDA for use in patients $>65$ years of age, who have complications such as coronary heart disease. It also increased the risk of water-sodium retention and cardiotoxicity. A comparison of the adverse drug reaction rate in the two arms also revealed a statistically significant difference, especially with regard to cardiotoxicity.

In China, patients usually go home after completing their chemotherapy course, so they usually do not comply well with blood routine examination requirements. Consequently, CIT cannot be detected and treated appropriately, causing a delay in the subsequent treatment, which may not be as effective. Our findings showed that the prophylactic administration of rhTPO and rhIL-11, which was suitable for Chinese patients, could guarantee the maintenance of scheduled dosage and the frequency of chemotherapy, with rhTPO being the most convenient platelet enhancer.

In conclusion, ours is the first comparative clinical study into the prophylactic administration of rhTPO and rhIL-11 for the treatment of CIT in NSCLC patients worldwide. Our findings suggest that the prophylactic administration of rhTPO helps to alleviate CIT in NSCLC as well as rhIL-11, but is more safe and convenient to use. It is also necessary to optimize further the rhTPO prophylactic administration schedule for CIT for the treatment of different solid tumors and chemotherapy regimens.

\section{Abbreviations}

rhTPO: recombinant human thrombopoietin; rhIL-11: recombinant human interleukin 11; CIT: chemotherapy-induced thrombocytopenia; NSCLC: non-small cell lung cancer; CFDA: China Food and Drug Administration; ECOG PS: Eastern Cooperative Oncology Group performance status; DIC: disseminated intravascular coagulation; CNS: central nervous system; GC: gemcitabine/cisplatin; GP: gemcitabine/ carboplatin; ECG: electrocardiograph; CI: confidence interval; VTE: venous thrombosis embolism.

\section{Acknowledgements}

The authors thank the following participating centers: Yanru Qin, Department of oncology, the First Affiliated Hospital of Zhengzhou University; Haiyan Liu, Department of Hematology and Oncology, General Hospital of Jilin Oil Field. Yiping Zhang, Department of oncology, Zhejiang Cancer Hospital; 
Tienian Zhu, Department of oncology, Bethune International Peace Hospital of The People's Liberation Army; Wei Liu, Department of oncology, Hebei Cancer Hospital; Xiaoling Li, Department of pneumology medical oncology, Liaoning Cancer Hospital. We express great thanks to the support from Shenyang 3S BIO Incorporation.

\section{Funding}

This work was supported by grants from the National Key R\&D Program of China (2016YFC13 03300), the National Natural Science Foundation of China (81672272), the Science and Technology Commission of Shanghai Municipality (STCSM) (16140902800), the Key Project of Shanghai Health \& Family Planning Commission (201540365), the Shenyang 3S BIO TCP young and middle-aged scientist research fund, and Shanghai Jiao Tong University School of Medicine Collaborative Innovation Center for Translational Medicine (TM201610).

\section{Informed consent}

Written informed consent was obtained from all participants included in the study.

\section{Competing Interests}

The authors have declared that no competing interest exists.

\section{References}

1. Torre LA, Bray F, Siegel RL, Ferlay J, Lortet-Tieulent J, Jemal A: Global cancer statistics, 2012. CA Cancer J Clin 2015, 65(2):87-108.

2. Kun Chen QL, Chunlin Chang, Yannan Zhao, Yue'e Liu, Na Wang, Huiling $\mathrm{Su}$, Yuehua Huang: A clinical comparative study of GP and TP 1st-line chemotherapies for advanced non-small cell lung cancer. The Chinese-German Journal of Clinical Oncology 2010, 9(11):621-624.

3. Jiang J, Liang X, Zhou X, Huang R, Chu Z, Zhan Q: Paclitaxel plus platinum or gemcitabine plus platinum in first-line treatment of advanced non-small-cell lung cancer: results from 6 randomized controlled trials. Int J Clin Oncol 2013, 18(6):1005-1013.

4. Hu X, Jiao S, Zhang S, Wang Z, Wang M, Huang C, Zheng R, Li K, Wang J, Wang $Y$ et al: [Efficacy and toxicity of pemetrexed or gemcitabine combined with cisplatin in the treatment of patients with advanced non-small cell lung cancer]. Zhongguo Fei Ai Za Zhi 2012, 15(10):569-575.

5. Wu Y, Aravind S, Ranganathan G, Martin A, Nalysnyk L: Anemia and thrombocytopenia in patients undergoing chemotherapy for solid tumors: a descriptive study of a large outpatient oncology practice database, 2000-2007. Clin Ther 2009, 31 Pt 2:2416-2432.

6. Ma J: Expert consensaus on rational use of recombinant human IL-11 for thrombocytopenia in solid tumor patients. Chinese Journal of Oncology 2010, 32:948-950.

7. Ballmaier M, Germeshausen M, Krukemeier S, Welte K: Thrombopoietin is essential for the maintenance of normal hematopoiesis in humans: development of aplastic anemia in patients with congenital amegakaryocytic thrombocytopenia. Ann N Y Acad Sci 2003, 996:17-25.

8. Kuter DJ: Managing thrombocytopenia associated with cancer chemotherapy. Oncology (Williston Park) 2015, 29(4):282-294.

9. Kuter DJ, Begley CG: Recombinant human thrombopoietin: basic biology and evaluation of clinical studies. Blood 2002, 100(10):3457-3469.

10. Chinese Society of Clinical O: [Expert consensus on diagnosis and treatment of chemotherapy-induced thrombocytopenia in cancer patients (2014 version]. Zhonghua Zhong Liu Za Zhi 2014, 36(11):876-879.

11. Ballmaier M, Germeshausen M, Schulze H, Cherkaoui K, Lang S, Gaudig A, Krukemeier S, Eilers M, Strauss G, Welte K: c-mpl mutations are the cause of congenital amegakaryocytic thrombocytopenia. Blood 2001, 97(1):139-146.

12. Kaushansky K: Historical review: megakaryopoiesis and thrombopoiesis. Blood 2008, 111(3):981-986.

13. Vadhan-RajS, Verschraegen CF, Bueso-Ramos C, Broxmeyer HE, Kudelka AP, Freedman RS, Edwards CL, Gershenson D, Jones D, Ashby $M$ et al:
Recombinant human thrombopoietin attenuates carboplatin-induced severe thrombocytopenia and the need for platelet transfusions in patients with gynecologic cancer. Ann Intern Med 2000, 132(5):364-368.

14. Xu YH, Cheng BJ, Lu S, Jian H, Zhou Z, Chen ZW, Ye XY: [Short-term intermittent prophylactic administration of recombinant human thrombopoietin attenuates chemotherapy-induced thrombocytopenia in lung cancer patients]. Zhonghua Zhong Liu Za Zhi 2011, 33(5):395-399.

15. Chinese Society of Clinical O: [Expert consensus on diagnosis and treatment of chemotherapy-induced thrombocytopenia in cancer patients (2014 version]. Zhonghua Zhong Liu Za Zhi 2014, 36(11):876-879.

16. Vadhan-Raj S: Recombinant human thrombopoietin in myelosuppressive chemotherapy. Oncology (Williston Park) 2001, 15(7 Suppl 8):35-38.

17. Shimazaki C, Inaba T, Uchiyama H, Sumikuma T, Kikuta T, Hirai H, Sudo $Y$, Yamagata N, Ashihara E, Goto $\mathrm{H}$ et al: Serum thrombopoietin levels in patients undergoing autologous peripheral blood stem cell transplantation. Bone Marrow Transplant 1997, 19(8):771-775 